\title{
GESTÃO SUSTENTÁVEL NA CADEIA DE SUPRIMENTOS DA INDÚSTRIA DO TABACO
}

\author{
${ }^{1}$ Gualter Baptista Junior \\ ${ }^{2}$ Heron Sergio Moreira Begnis
}

\begin{abstract}
RESUMO
O sucesso empresarial não se resume aos resultados financeiros, mas também pondera sobre a responsabilidade socioambiental, compreendendo a visão estratégia, por vezes de sobrevivência, interessando às organizações da cadeia de suprimentos, contemplar competitividade e sustentabilidade. Tal intenção é ainda mais desafiadora em setores econômicos considerados controversos. Partindo deste entendimento, esse artigo tem como objetivo investigar a ação estratégica da Empresa Focal para o desenvolvimento da gestão sustentável da cadeia de suprimentos do tabaco em folha. Para alcançar este objetivo, foi desenvolvido um estudo de caso centralizado na perspectiva de uma Empresa Focal representativa do setor. A análise está sustentada na perspectiva do tripé da sustentabilidade (Tripple Bottom Line - TBL) e da abordagem da gestão sustentável das cadeias de subimentos (GSCS). Como resultado, observou-se o desnivelamento informativo referente às práticas adotadas pela Empresa Focal ao longo de sua cadeia de suprimentos, especialmente junto aos fornecedores de suprimentos críticos, que demonstram não compreender a magnitude da sustentabilidade e sua implicação estratégica.
\end{abstract}

Palavras-chave: Cadeias de suprimentos. Gestão sustentável. Sustentabilidade. Tabaco.

\section{TOBACCO INDUSTRY SUPPLY CHAIN SUSTAINABLE MANAGEMENT}

\begin{abstract}
Business success is not just about financial results. It considers socio-environmental responsibility and understands a strategic vision, sometimes of survival and imposing on the supply chain to contemplate competitiveness and sustainability together. This intention is even more challenging in controversial economic sectors. This understanding leads the article to investigate the strategic action of the Focal Company for the development of sustainable management of the leaf tobacco supply chain. A case study was carried out from the perspective of a Focal Company representing the sector to achieve this objective. The analysis is supported by the perspective of the Tripple Bottom Line - TBL and the approach of Sustainable Supply Chain Management (SSCM). As a main conclusion, the misalignment of information regarding the practices adopted by the Focal Company throughout its supply chain was evidenced. The misalignment is especially verified with suppliers that demonstrate not understanding the magnitude of sustainability and its strategic implementation.
\end{abstract}

Key words: Supply chains. Sustainable management. Sustainability. Tobacco.

\footnotetext{
${ }^{1}$ Universidade de Santa Cruz do Sul - UNISC, Rio Grande do Sul, (Brasil). https://orcid.org/0000-0001-9030-7070 E-mail: https://orcid.org/0000-0001-9030-7070

${ }^{2}$ Universidade de Santa Cruz do Sul - UNISC, Rio Grande do Sul, (Brasil). https://orcid.org/0000-0002-0561-7590 E-mail: heron@unisc.br
} 


\section{INTRODUÇÃO}

$\mathrm{O}$ crescimento industrial alcançou escalas globais durante o século XX e trouxe um conjunto de externalidades intrínsecas, como impacto ambiental e social, resultando, muitas vezes, em danos e destruição. Talvez a principal distinção entre o século XX e o século XXI esteja centrada justamente na preocupação das repercussões ambientais e sociais da ação empresarial. Uma das principais diferenças do pensamento das atuais gerações em relação às que a antecederam é a consciência de que ações e explorações humanas têm efeitos substanciais no futuro (Bossel, 1987). Muito provavelmente esta afirmação tenha mais sentido hoje, passados 30 anos do artigo de Bossel. Essa argumentação ganha força com o surgimento do conceito de "sustentabilidade" no mainstream na década de 1980 (Purvis, Mao \& Robinson, 2019). A sustentabilidade significa transformar os modos de vida para maximizar as chances de que as condições ambientais e sociais apoiem indefinidamente a segurança humana, o bem-estar e a saúde (Daly, 1996).

Embora o pensamento sustentável apresente-se bem difundido no contexto individual (Polistina, 2018; Marcus \& Roy, 2019), é sabido que, na esfera organizacional, a incorporação das questões da responsabilidade ambiental e social ainda é uma incógnita. Quando tratada, concentrase no campo das questões econômicas. Parte disso pode estar associada às inúmeras críticas que o termo sustentabilidade recebeu na época após sua publicação no Relatório Brundtland (Brundtland, 1987). A principal crítica é em relação a definição do termo sustentável, que significa algo que pode se desenvolver e permanecer o mesmo (sustentável).

A partir disso, busca-se a criação de um modelo que possa tornar tangível às organizações empresariais o tema sustentabilidade. Neste viés, surgiu o Tripple Bottom Line - TBL (Elkington, 1994), arquétipo que define os três pilares da sustentabilidade: o econômico, com a criação de empreendimentos viáveis e atraentes aos investidores; o ambiental, com a interação de processos ambientais que não promovessem danos permanentes à natureza e, o social, com o estabelecimento de ações justas à trabalhadores, parceiros e sociedade. A base do TBL consiste no entendimento de que o sucesso empresarial não se relaciona somente às medidas financeiras tradicionais e sim pela ética, responsabilidade social e desempenho ambiental, fundamentando a mudança do paradigma empresarial focado no lucro (Barbieri \& Cajazeira, 2010).

A responsabilidade socioambiental deixou de ser opção para tornar-se questão de visão estratégica e, por vezes, de sobrevivência (Porter \& Kramer, 2002; Blasi, Caporin \& Fontini, 2018). Paralelamente ao processo, transcorreu o marco conceitual da eclosão da sustentabilidade: a alteração do perfil mercadológico do consumidor, este, tornando-se mais participativo ao modelo de negócios empresariais, exigindo novos padrões de conduta fiscalizando e determinando postura à operacionalização (Baldassarre \& Campo, 2016; Fargnoli et al., 2018).

Nesta esfera, as organizações passam a interagir com demais agentes sociais visando a sobrevivência, assumindo relevante papel de responsabilidade social corporativa e de desenvolvimento econômico, convergindo à inclusão social, diversidade e diminuição da pobreza (Carroll, 1999; Lee, 2008; Vollero et al., 2019). Assim, é interessante às organizações a implementação de ações voltadas à sustentabilidade e que estas se estendam ao longo de sua cadeia de suprimentos. Percebe-se uma transformação nas estratégias organizacionais no tocante à necessidade de gestão e ao fortalecimento da cadeia de suprimentos de inserção, haja vista a sociedade, principalmente, consumidores, importarem-se cada vez mais com a origem e aos processos pelos quais passam os produtos e serviços ofertados (Vitari \& David, 2017; Barbieri \& Cajazeira, 2010).

Enquanto o conceito de sustentabilidade e a lógica do TBL destacam que é necessário expandir a atenção da gestão para além de suas fronteiras organizacionais (Erekson, Loucks \& Aldag, 1994), o próprio conceito de gestão das cadeias de suprimentos - GCS (Cooper, Lambert \& Pagh, 1997) passa a ser expandido e complementado por uma nova perspectiva: a Gestão Sustentável da Cadeia de Suprimentos - GSCS (Beske \& Seuring, 2014; Rajeev et al., 2017). Assim, a gestão sustentável da cadeia de suprimentos se refere ao conjunto de organizações 
conectadas e interdependentes que, frente à cooperação mútua, controlam, gerenciam e aperfeiçoam o fluxo de matérias-primas e informação para o cliente final, gerando com isso, competitividade e sustentabilidade aos negócios (Christopher, 2007; Carter \& Rogers, 2008; Seuring \& Müller, 2008; Shokri Kahi, 2017; Qorri, Mujkić \& Kraslawski, 2018).

Neste contexto, este artigo tem o objetivo de investigar a ação estratégica da Empresa Focal (Lambert, Cooper \& Pagh, 1998) para o desenvolvimento da Gestão Sustentável da Cadeia de Suprimentos (Carter \& Rogers, 2008; Seuring \& Müller, 2008) do tabaco em folha sob a perspectiva do Triple Bottom Line (TBL). Na cadeia do tabaco em folha, a integração das empresas possibilita aos produtores rurais acesso a meios modernos de produção, tecnologias, incentivos à continuidade da cultura por meio de garantia de comercialização da produção de tabaco, assistência técnica e financeira (Begnis, Arend \& Alievi, 2017). Enquanto campo de estudo para a gestão sustentável da cadeia de suprimentos, a indústria do tabaco apresenta características relevantes por estar inserida em um mercado controverso (Palazzo \& Richter, 2005; Aqueveque, Rodrigo \& Duran, 2018). Estes são mercados legais e legítimos (Vollero et al, 2019) que são envolvidos por questões morais, religiosas, de saúde, prejudiciais, perigosas, com impacto ambiental ou socialmente discriminatórias, como o representado pela indústria do petróleo (De Roeck \& Delobbe, 2012; Du \& Vieira, 2012); cassinos e loterias e pela indústria de bebidas alcoólicas (Jo \& Na, 2012; Grougiou, Dedoulis \& Leventis, 2016).

Para alcançar o objetivo proposto, foi desenvolvido um estudo de caso com uma das maiores empresas multinacionais do segmento. Este artigo inicia, após esta introdução, com a apresentação da base teórica, que inclui a incorporação do conceito de sustentabilidade no contexto empresarial, especialmente nas cadeias de suprimentos, e a construção do entendimento acerca da gestão sustentável das cadeias de suprimentos. Na sequência, o texto traz o detalhamento da metodologia empregada e a discussão dos resultados, finalizando com as conclusões.

\section{REFERENCIAL TEÓRICO}

Esse artigo é sustentando por dois aportes teóricos. O primeiro, diz respeito a sustentabilidade, mais especificamente envolvendo a perspectiva do Triple Bottom Line - TBL ou também chamado tripé da sustentabilidade (Elkington; 1994, 2012). O segundo, se trata da evolução do conceito de Gestão da Cadeia de Suprimentos - GCS (Cooper, Lambert \& Pagh, 1997; Christopher, 2007) para o de Gestão Sustentável da Cadeia de Suprimentos - GSCS (Carter \& Rogers, 2008; Seuring \& Müller, 2008; Shokri Kahi, 2017; Qorri, Mujkić \& Kraslawski, 2018).

\subsection{Sustentabilidade: o Triple Bottom Line}

O tema da sustentabilidade vem ganhando destaque em diversas áreas de pesquisa (Lis, Sudolska \& Tomanek, 2020), no entanto, boa parte das publicações sobre o tema se concentra nos aspectos teóricos e conceituais (Khan et al., 2020). Cada vez mais se exige das organizações empresariais uma atuação alinhada ao conceito de desenvolvimento sustentável. Essa exigência obriga as organizações a repensarem seu modelo de negócio com vistas a permitir aos seus clientes suprir suas necessidades ao menor custo socioambiental (Chang, Chiang \& Pai, 2012; Vandchali, Cahoon \& Chen, 2021), o que implica em novas definições sobre a missão organizacional e os modelos de negócios (Hoffman, 2018; Good \& Thorpe, 2020). As pressões externas impõem que a lógica da sustentabilidade seja incorporada às ações organizacionais (Simões-Coelho \& Figueira, 2021). O desafio enfrentado por organizações individuais é o de descobrir novas maneiras de cooperar com seus fornecedores, clientes e outros stakeholders, garantindo ao mesmo tempo que as organizações se beneficiem não apenas em termos de cidadania corporativa, mas também em termos de vantagem competitiva (Elkington; 1994) e aumento da satisfação dos clientes (Belas et al., 2021). 
John Elkington, no final da década, apresenta o conceito Triple Bottom Line ou Tripé da Sustentabilidade. Sua proposta principal é a mensuração dos resultados de uma organização partir dos pilares econômico, social e ambiental (Elkington; 2012) e a clareza do conceito de sustentabilidade é alcançada a partir da estruturação destes três pilares (Barbieri \& Cajazeira, 2010). Deste modo, a sustentabilidade envolve a atividade economicamente viável, socialmente justa e ecologicamente correta, implicando em uma nova cultura corporativa (Solana-Ibáñez, CaravacaGarratón \& Teruel-Sánchez, 2020).

O pilar social se ocupa de todo capital humano, direta ou indiretamente, relacionado às atividades desenvolvidas por uma empresa, incluindo colaboradores, público-alvo, fornecedores e a sociedade em geral. Diz respeito aos valores sociais e culturais e à justiça na distribuição de custos e benefícios. O pilar econômico centra-se em uma empresa economicamente sustentável. Desta forma, a empresa deve ser capaz de produzir, distribuir e oferecer produtos ou serviços estabelecendo uma relação de competitividade justa em relação aos concorrentes. A perspectiva econômica resulta da aquisição de rendimento suficiente ao custeio da vida em sociedade. No tocante ao aspecto ambiental, o desenvolvimento sustentável se refere às condutas que possuam, direta ou indiretamente, impacto sobre o meio ambiente, a curto, médio ou longo prazo, buscando minimizar os impactos ambientais causados pela produção industrial. A empresa que atua alinhada ao modelo TBL está orientada a buscar resultados positivos nestas três esferas propostas por Elkington (2012).

Assim, impõe-se às empresas a geração de inovações de produto, serviço, processo que compatibilizem o impacto da produção e consumo com o provimento de recursos naturais renováveis e não-renováveis, com a assimilação dos resíduos e da poluição decorrente, tendo em vista a manutenção dos serviços ambientais essenciais à vida. Nesta perspectiva, o desempenho organizacional é avaliado não apenas pelo valor econômico, mas conjuntamente pelo valor social e ambiental (Elkington, 2012; Schaltegger, Hörisch \& Freeman, 2019; Blasi, Caporin \& Fontini, 2018; Ho \& Lucianetti, 2021).

A aplicação do conceito TBL em uma organização não é uma tarefa fácil. A complexidade aumenta quando a organização transborda sua missão de organização sustentável para sua cadeia de suprimentos. Caso a organização ocupe o papel central nessa cadeia, o papel de empresa focal, ocorre o transbordamento, o que confere ainda mais responsabilidade à empresa focal, amplia a atuação, redesenha processos e acentua os compromissos envolvidos. Para entender melhor a problemática apresentada, a seguir, será discutida a gestão sustentável da cadeia de suprimentos.

\subsection{Gestão sustentável da cadeia de suprimentos}

Cadeias de suprimentos são compostas por agentes que atuam ativamente no atendimento da demanda do mercado consumidor, disponibilizando produtos e serviços em quantidade, qualidade e preço esperados como também, atuando enquanto estimuladores da demanda dos produtos (Talamini, Pedrozo \& Silva, 2005). A dimensão dessa cadeia define-se pela quantidade de membros que a complexidade do processo produtivo demanda para realização. Contudo, nem todos os membros dessa cadeia possuem a mesma importância sob o ponto de vista do seu gerenciamento (Maixel \& Luoma, 2015).

A cadeia de suprimentos deveria ser entendida como uma entidade única, tendo em vista as responsabilidades compartilhadas e colaborativas entre os membros, que devem emparelhar os objetivos em todos os níveis, tomando decisões estratégicas em função do impacto sobre os custos totais, a participação no mercado e as mudanças no ambiente competitivo. Todavia, tais fundamentos somente são possíveis pela Gestão da Cadeia de Suprimentos - GCS ou Supply Chain Management - SCM. Nesse sentido, a GCS compreende o conjunto de abordagens utilizadas na integração e sincronia eficientes dos participantes no contexto das mercadorias e serviços serem produzidos, distribuídos e consumidos na quantidade, qualidade, localização e tempo certo para 
minimizar custos globais dos sistemas no tempo em que alcancem o nível de serviço desejado (Ageron, Gunasekaran \& Spalanzani, 2012).

A visão da gestão da cadeia de suprimentos, referindo-se à gestão de fluxos de materiais entre organizações, foi representada em duas definições intercorrentes ao final da década de 1990, sendo que a primeira, de Monczka, Trent \& Handfield (1998, p. 12), estabelece o conceito "[...]cujo objetivo principal é integrar e gerenciar a origem, fluxo e controle de materiais por meio de uma perspectiva sistêmica ao longo de múltiplas funções e múltiplos níveis de fornecedores". Já a segunda, de Handfield e Nichols (1999, p. 17), engloba todas as atividades "[...] associadas com o fluxo e transformação de bens desde o estágio de matérias primas (extração) até o consumidor final, assim como os fluxos de informação associados".

A compreensão de como as empresas se posicionam na estrutura e como estabelecem os diversos vínculos são aspectos essenciais para o entendimento da dinâmica da Gestão da Cadeia de Suprimentos (Chia, 2014). Tendo em vista o poder de influenciar a cadeia a qual pertence, a Empresa Focal é fundamental na introdução de novas práticas sustentáveis entre os agentes da cadeia a qual está inserida. A Empresa Focal é aquela que é capaz de estabelecer a governança em uma cadeia de suprimentos, induzindo a cooperação entre os diversos e diferentes atores (Seuring \& Müller, 2008; Lambert, Cooper \& Pagh, 1998; Beske \& Seuring, 2014). Mecanismos de governança na GSCS são fundamentais para alcançar resultados sustentáveis (Koberg \& Longoni, 2019; Jia, Gong \& Brown, 2019) e reduzir os riscos (Gouda \& Saranga, 2018; Chowdhury \& Quaddus, 2021). O poder da Empresa Focal em relação a sua capacidade de influência pode estar ligado a diversos fatores, tais como estrutura do seu setor econômico, grau de concentração, escala, barreiras à entrada, entre outros, além de estabelecimento de incentivos apropriados à cooperação e introduzindo inovações que agreguem valor ao longo da cadeia (Carvalho \& Barbieri, 2013). Os gestores da empresa focal desempenham um papel ativo na gestão da sustentabilidade dos fornecedores, informando sobre as políticas de sustentabilidade e monitorando as desconformidades identificadas por auditores terceirizados (Xiao et al., 2019).

Percebe-se que, além da demanda por um desempenho econômico satisfatório, as organizações passaram a ser responsabilizadas, também, por um desempenho social e ambiental positivo em relação às suas operações $\mathrm{e}$, indiretamente, às atividades de seus parceiros $\mathrm{e}$ fornecedores. Assim, a atual atenção voltada às cadeias de suprimentos está relacionada ao gerenciamento de processos mais eficientes, inovadores e sustentáveis (Schulz et al., 2016). Com a crescente demanda por integrar questões ambientais e sociais à gestão da cadeia de suprimentos, percebeu-se o surgimento de conceitos de sustentabilidade relacionando-se à gestão operacional da cadeia de suprimentos (Jia, Gong \& Brown, 2019), tanto em razão dos recursos finitos como pela forma de processamento de produtos e serviços (McWilliams et al., 2016). Inicialmente a gestão da cadeia de suprimentos focava em questões relacionadas à integração de processos entre parceiros da cadeia, análise de custo-eficiência dos fornecedores e serviços aos consumidores. Contudo, discussões socioambientais associadas aos questionamentos sobre impactos de produção e consumo despontaram novos interesses: logística reversa, gestão ambiental e cadeia de suprimento verde e sustentável. Assim, percebe-se que a gestão sustentável de operações aproximou a visão tradicional da gestão (lucro e eficiência) aos aspectos de impactos ao meio ambiente e aos públicos de interesse (McWilliams et al., 2016).

Assim, são criadas oportunidades de incremento da competitividade empresarial advinda do alinhamento entre ações de responsabilidade social e estratégia organizacional (Schulz et al., 2016; Khodakarami, Petersen \& Venkatesan, 2015). Nesse sentido, Barbieri e Cajazeira (2010) colocam a convergência dos movimentos a favor da responsabilidade social empresarial e da sustentabilidade empresarial. A cadeia de suprimento sustentável é aquela na qual o relacionamento com fornecedores e outros agentes orienta-se ao desenvolvimento de inovações técnicas, culturais, de gestão e de processos (Carter \& Rogers, 2008; Seuring \& Müller, 2008) tendo em vista ultrapassar as questões ambientais nas cadeias de suprimento (Souza \& Ribeiro, 2013). A implementação desse objetivo é um desafio significativo para os gestores que gerenciam as cadeias de suprimentos, pois 
requer consistência, criatividade, o emprego de um volume significativo de recursos e, acima de tudo, a consideração das necessidades dos principais stakeholders que compõem o ambiente de negócios (Zimon, Tyan, \& Sroufe, 2020).

Mais recentemente, o campo de pesquisa em sustentabilidade em cadeias de suprimento tem evoluído de uma perspectiva isolada das interfaces para uma visão de responsabilidade social empresarial aplicada à cadeia de suprimento, aferindo o início do processo de convergência da abordagem TBL como perspectiva de sustentabilidade que contribui à emergência da Gestão Sustentável da Cadeia de Suprimentos - GSCS como um modelo teórico. Não obstante, a GSCS conduziu à expansão das fronteiras contemplando processos não anteriormente considerados pela GCS (Shokri Kahi, 2017; Qorri, Mujkić \& Kraslawski, 2018).

A Gestão Sustentável da Cadeia de Suprimentos demanda a compreensão da difusão das boas práticas ambientais, além da amplificação dos impactos ambientais negativos decorrentes do modelo de cadeia de suprimento cuja preocupação atende ao cliente sem se importar com os danos causados ao meio ambiente (Srivastava, 2007). Em cadeias de suprimento globais, “[...] o sucesso da empresa estendida é positivo apenas para as receitas da organização, porém negativo para o meio ambiente" (Halldórsson et al., 2009, p. 91). Os mesmos autores colocam que a implantação de melhorias em práticas socioambientais na Gestão da Cadeia de Suprimentos demanda maior responsabilidade tanto em relação ao ciclo de vida dos produtos quanto às condições de trabalho, remuneração e respeito aos direitos essenciais do ser humano (Halldórsson et al., 2009).

Ansari e Kant (2017) apontam as barreiras à implementação da gestão sustentável da cadeia de suprimentos, tais como falta de informação e transparência, falta de treinamento e experiência, competências de fornecedores, implicações de custos, falta de compromisso de alta gerência, falta de recursos financeiros, complexo em design para reduzir o consumo de recursos e energia, facilidade inadequada para adoções de práticas logísticas reversas e a falta de implementação de tecnologias de informação - TI. Implicações de custos, falta de compromisso com a alta administração, falta de treinamento e experiência e compromisso de fornecedores de baixo desempenho são as barreiras mais comuns consideradas à implementação bem-sucedida da gestão sustentável da cadeia de suprimentos. Sajjad, Eweje e Tappin (2020) reforçam que preocupações com custos, restrições estratégicas e estruturais, bem como problemas com fornecedores e clientes e a falta de regulamentações eficazes representam obstáculos críticos para a implementação da GSCS.

O objetivo principal a ser atingido pela GSCS é ampliar cada vez mais o valor percebido dos produtos que serão entregues ao consumidor final. A percepção do valor pelos consumidores está intimamente ligada aos atributos que estes mais valorizam. Neste cerne, a Empresa Focal contribui para a gestão sustentável quando sua atuação gera resultados positivos em termos econômicos, sociais e ambientais que se estendem e estão coordenados ao longo de toda a sua cadeia de suprimentos. Isto implica na necessidade de articulação e colaboração entre os componentes da cadeia de suprimentos. Em seus diferentes níveis de profundidade, esta articulação visa melhorar a relação entre os elos da cadeia e, efetivamente, contribuir com a introdução de práticas sustentáveis (Alves, Silva \& Santos, 2018).

\section{MÉTODO}

Esta pesquisa emprega a abordagem qualitativa e pode ser caracterizada como um estudo de natureza exploratório e descritivo. Como estratégia metodológica, empregou-se o estudo de caso porque possibilita a análise mais abrangente do problema de pesquisa, uma vez que tratar das estratégias de Gestão Sustentável da Cadeia de Suprimentos exige uma abordagem holística.

A escolha da cadeia de suprimentos do tabaco como objeto da pesquisa (caso) deve-se ao fato de a mesma estar consolidada na região Sul do Brasil, além de ter atuação globalizada, isto é, a cadeia de suprimentos do tabaco atua em mais de 150 países, operada por grandes conglomerados globais cujo mercado atinge quase 2 bilhões de pessoas ao redor do mundo. Além disso, por ser um 
setor altamente controverso, pode ser compreendido como um caso extremo (Yin, 2015). Já a definição da Empresa Focal para a realização da pesquisa deu-se de maneira intencional, tendo em vista ser pioneira no setor em que atua, ter uma história de sucesso em seus mais de 115 anos de existência, além de liderar os processos de mudanças no setor, sendo referência para diversas empresas do agronegócio.

A coleta dos dados se baseou nas informações fornecidas pelos principais stakeholders e componentes da cadeia de suprimentos da Empresa Focal (Figura 1). A coleta de dados foi efetuada por meio de entrevistas abertas em profundidade e outras fontes de informações relacionadas à Empresa Focal, tais como site institucional, Relatório Anual, Relatório de Sustentabilidade 2015 e 2016, Termo de Cessação de Conduta, Portal do Produtor de Tabaco e diversas publicações organizacionais.

Inicialmente foram realizadas entrevistas com oito gestores da Empresa Focal com o propósito central de identificar a visão da gestão em relação às estratégias e ações por meio das quais a Empresa Focal trata da sustentabilidade. Após as entrevistas junto à Empresa Focal, foram entrevistados três gestores de empresas globais fornecedoras de insumos para a produção de tabaco com o objetivo de avaliar a relação com a Empresa Focal e suas percepções sobre as estratégias inerentes a sustentabilidade. Também foram entrevistados dois principais clientes (mercado nacional e internacional) da Empresa Focal, buscando coletar suas visões sobre a Empresa Focal no que tange a sustentabilidade da cadeia do tabaco. Adicionalmente foram entrevistados seis produtores de tabaco integrados pela Empresa Focal, sendo dois produtores no Paraná, dois em Santa Catarina e dois no Rio Grande do Sul com intuito também de analisar quais as percepções dos produtores integrados com relação a Empresa Focal e suas estratégias de sustentabilidade para a cadeia de suprimentos. As entrevistas foram realizadas no período que se estendeu entre dezembro de 2018 a janeiro de 2019, sendo gravadas e transcritas.

\begin{tabular}{|c|l|c|}
\hline Segmento & \multicolumn{1}{|c|}{ Descrição do respondente } & Código \\
\hline & Gerente Nacional de Produção Agrícola & EMF 01 \\
& Gerente de Sustentabilidade e Relacionamento com Produtores & EMF 02 \\
& Gerente de Difusão de Tecnologias & EMF 03 \\
& Gerente Territorial de Produção Agrícola & EMF 04 \\
& Gerente Regional de Produção Agrícola & EMF 05 \\
& Gerente de Serviços e Informações de Produção Agrícola & EMF 06 \\
& Coordenador de Produção Agrícola & EMF 07 \\
& Especialista em Logística & EMF 08 \\
\hline \multirow{2}{*}{ Clientes } & Senior Sourcing Manager & CLI 01 \\
& Gerente de Planejamento e Blending & CLI 02 \\
\hline \multirow{2}{*}{ Fornecedores de } & Diretor Geral - Segmento de Substrato & FOR 01 \\
Insumos & Superintendente - Segmento de Fertilizantes & FOR 02 \\
& Representante Técnico de Vendas - Segmento Agroquímicos & FOR 03 \\
\hline \multirow{3}{*}{ Produtores de } & Fornecedor a 4 anos da Empresa Focal - Paraná & PRO 01 \\
Tabaco & Fornecedor a 25 anos da Empresa Focal - Paraná & PRO 02 \\
& Fornecedor a 8 anos da Empresa Focal - Santa Catarina & PRO 03 \\
& Fornecedor a 25 anos da Empresa Focal - Santa Catarina & PRO 04 \\
& Fornecedor a 20 anos da Empresa Focal - Rio Grande do Sul & PRO 05 \\
& Fornecedor a 27 anos da Empresa Focal - Rio Grande do Sul & PRO 06 \\
\hline
\end{tabular}

Figura 1. Agentes entrevistados e descrição dos respondentes

Fonte: Dados da pesquisa

As entrevistas em profundidade seguiram um roteiro constituído de três blocos, sendo eles: estratégias interorganizacionais, sustentabilidade e gestão sustentável. Este roteiro foi submetido a um painel de especialistas composto por profissionais e acadêmicos da área que fizeram apontamentos que foram incorporados no roteiro final das entrevistas. 
Após a coleta de dados, aplicou-se a técnica de análise de conteúdo que, consoante Mozzato e Grzybovski (2011), tem por objetivo ultrapassar incertezas enriquecendo os dados coletados, visto entender criticamente o sentido dessas comunicações, seu conteúdo e as significações explícitas ou ocultas. Nesta pesquisa, tal análise partiu de três categorias principais desenvolvidas a partir do referencial teórico apresentado na seção anterior e sintetizadas no Figura 2.

\begin{tabular}{|c|c|c|}
\hline Categorias principais & $\begin{array}{l}\text { Categorias } \\
\text { secundárias }\end{array}$ & Base teórica \\
\hline \multirow{3}{*}{$\begin{array}{l}\text { Estratégias } \\
\text { organizacionais e } \\
\text { interorganizacionais }\end{array}$} & $\begin{array}{l}\text { Motivação } \\
\text { estratégica }\end{array}$ & $\begin{array}{l}\text { Beske \& Seuring (2014); Carvalho \& Barbieri (2013); Good \& Thorpe } \\
\text { (2020); Hoffman (2018) }\end{array}$ \\
\hline & $\begin{array}{l}\text { Relações com } \\
\text { clientes }\end{array}$ & $\begin{array}{l}\text { Baldassarre \& Campo (2016); Fargnoli et al. (2018); Belas et al. } \\
\text { (2021); Simões-Coelho \& Figueira (2021); Vitari \& David (2017) }\end{array}$ \\
\hline & $\begin{array}{l}\text { Relações com } \\
\text { fornecedores }\end{array}$ & $\begin{array}{l}\text { Alves, Silva \& Santos (2018); Belas et al. (2021); Chang, Chiang \& } \\
\text { Pai (2012); Chiang \& Pai (2012); Jia, Gong \& Brown (2019); } \\
\text { Vandchali, Cahoon \& Chen (2021); Xiao et al. (2019) }\end{array}$ \\
\hline \multirow{3}{*}{ Sustentabilidade } & Econômica & \multirow{3}{*}{$\begin{array}{l}\text { Barbieri \& Cajazeira (2010); Brundtland (1987); Carter \& Rogers } \\
\text { (2008); Daly (1996); Elkington (1994); Elkington (2012); Jia, Gong \& } \\
\text { Brown (2019); Purvis, Mao \& Robinson (2019); Simões-Coelho \& } \\
\text { Figueira (2021); Vollero et al. (2019) }\end{array}$} \\
\hline & Social & \\
\hline & Ambiental & \\
\hline \multirow{4}{*}{ Gestão sustentável } & Barreiras & $\begin{array}{l}\text { Ansari \& Kant (2017); Sajjad, Eweje \& Tappin (2020); Zimon, Tyan, } \\
\text { \& Sroufe (2020) }\end{array}$ \\
\hline & Recursos & $\begin{array}{l}\text { Ansari \& Kant (2017); Maixel \& Luoma (2015); Seuring \& Müller } \\
\text { (2008) }\end{array}$ \\
\hline & $\begin{array}{l}\text { Avaliação e } \\
\text { desempenho }\end{array}$ & $\begin{array}{l}\text { Barbieri \& Cajazeira (2010); Blasi, Caporin \& Fontini (2018); Chan \& } \\
\text { Qi (2003); Ho \& Lucianetti (2021); Schulz et al. (2016) }\end{array}$ \\
\hline & Risco & $\begin{array}{l}\text { Beske \& Seuring (2014); Chowdhury \& Quaddus (2021); Gouda \& } \\
\text { Saranga (2018) }\end{array}$ \\
\hline
\end{tabular}

Figura 2. Categorias de análise

Fonte: Elaborado pelos autores

\section{RESULTADOS E DISCUSSÕES}

Os resultados da pesquisa são apresentados nesta seção, na qual são identificadas as estratégias da Empresa Focal voltadas à garantia da sustentabilidade e apontados gargalos que dificultam a sustentabilidade na Cadeia de Suprimentos do Tabaco. Por fim, estes resultados são discutidos com base no referencial teórico que dá suporte à pesquisa.

\subsection{Estratégias organizacionais e interorganizacionais}

O setor do tabaco atua, de forma constante, em programas de gestão socioambiental, recentemente, destaca-se o Sustainable Tobacco Programme (STP), este, distribuído nas áreas de Agronomia e Processamento, reúne 70 princípios e 175 critérios que estabelecem indicadores à produção de tabaco sustentável. No caso da Empresa Focal, esta, produziu uma cartilha tangente ao STP, distribuindo aos produtores integrados e aos demais stakeholders, além de publicar trimestralmente a revista "O Produtor Integrado de Tabaco", com informações de produção de tabaco sustentável.

Estas ações que visam a sustentabilidade da cadeia de suprimentos derivam do entendimento da Empresa focal de que é necessário gerar valor compartilhado junto com os stakeholders. Entendimento que é corroborado pelas considerações de um dos gestores da Empresa Focal (entrevistado EMF 04) ao expor que "[...]uma empresa que está no mercado a mais de 100 anos, que tem um sistema integrado de produção [...] está sempre pensando no futuro"; e ainda coloca o mesmo entrevistado "[...] os pilares econômico, social e ambiental são a chave do futuro [...] de sucesso, [...] sendo viável para a companhia, para todos os stakeholders e produtores [...]”. 
No tocante a como a empresa define e estrutura sua estratégia de sustentabilidade, outro gestor entrevistado (EMF 05) revelou que a Empresa Focal, alinhada "[...]às estratégias de sua Controladora internacional, revisou seu posicionamento estratégico [...], estruturando sua estratégia de sustentabilidade em três grandes áreas: (1) Agricultura sustentável; (2) Prosperidade no campo; (3) Redução de riscos".

As práticas sustentáveis estão na essência da empresa, faz parte de seu DNA, onde todas as
ações são amplamente difundidas, tanto interna como externamente, com isso todos os
envolvidos se sentem parte de todo o processo sustentável, sendo eles mesmos os
incentivadores destas práticas dentro e fora da organização. Não há um mecanismo de
premiação ou punição, há uma seleção natural de quem está utilizando essas práticas e
quem não está (EMF 08).

Neste sentido, múltiplas ações foram desenvolvidas buscando a deferência das estratégias contemporizando as premissas do $T B L$. Ao longo das etapas produtivas, a Empresa Focal utiliza tecnologia e controles, tendo em vista reduzir impacto no meio ambiente. Para isso, emprega ações estratégicas de neutralização de $83 \%$ das emissões de carbono e $98,12 \%$ de reciclagem dos resíduos sólidos gerados. Além disso, faz o tratamento de efluentes líquidos não coligados à rede municipal de esgoto, sendo reutilizada $100 \%$ da água dentro da própria fábrica e $99,6 \%$ dos resíduos sólidos gerados são reutilizados internamente em processos ou reciclados. Já no que diz respeito às matrizes energéticas, a Empresa Focal prioriza o uso de fontes renováveis, como a lenha utilizada, que é de madeira de reflorestamento e a sua frota vem sendo substituída gradativamente por veículos que utilizam combustíveis renováveis. Não obstante, a Empresa Focal investe no consumo sustentável das unidades fabris com sistemas de iluminação natural e aproveitamento da luz solar, reduzindo gastos com energia em uma área superior a $10.000 \mathrm{~m}^{2}$.

A Empresa Focal realiza ainda o monitoramento de emissão de gases de efeito estufa em sua cadeia produtiva, identificando áreas nas quais se pode adotar alternativas mais eficazes de redução das emissões e consequente aumento da eficiência energética. Adicionalmente, esta preocupação permite à Empresa Focal atestar, frente aos seus diversos stakeholders, o seu compromisso com a sustentabilidade.

Com relação a seus colaboradores, a Empresa Focal também adota os mais elevados padrões de comportamento empresarial e de transparência, respeitando sempre a legislação e as regulamentações aplicáveis ao negócio, além dos mais elevados padrões de ética empresarial. Para tanto, implementou, em 2016, o Programa de Integridade, tendo por pilares o comprometimento da alta direção; a divulgação de políticas internas claras; um canal de denúncia confiável; o monitoramento constante de riscos e implementação de controles apropriados e a comunicação e o treinamento de colaboradores e terceiros. Ainda, o Código de Conduta Ética compila todos os compromissos da empresa com o comportamento responsável, ético, transparente e de respeito mútuo entre colaboradores e fornecedores, orientando sobre práticas anticorrupção, conflitos de interesse, relacionamentos com fornecedores, entre outros.

Por meio dos apontamentos das entrevistas acerca da motivação estratégica da Empresa Focal, identifica-se que esta decorre dos valores organizacionais defendidos, tal como destacado pelos entrevistados: (EMF 02, CLI 01, FOR 01), transparência (FOR 01, PRO 02), responsabilidade social, econômica e ambiental (EMF 02, PRO 04), integridade (EMF 04); diversidade (EMF 07, PRO 06), sustentabilidade (EMF 07, CLI 02, FOR 03) e respeito (FOR 01, PRO 01). A definição e estruturação das estratégias de sustentabilidade estão pautadas nas diretrizes da OMS, IBAMA, MAPA (EMF 04, FOR 01) e do Grupo Controlador da Empresa Focal

[...]estruturando sua estratégia de sustentabilidade em três grandes áreas: (1) Agricultura sustentável; (2) Prosperidade no campo; (3) Redução de riscos" (EMF 05) neste sentido fomenta-se pelos "princípios de sustentabilidade financeira da cadeia, preservação ambiental, promoção sociocultural, garantia de suprimento no longo prazo (parceria) e qualidade superior de produto" (CLI 02), tudo isso "através de clareza ou idoneidade" (CLI 01). 


\subsection{Sustentabilidade}

No tocante à adoção de estratégias voltadas à sustentabilidade econômica, social e ambiental, expõem os entrevistados que a Empresa focal assim as faz "[...]por que entende que esses são os principais pilares da sustentabilidade e prosperidade" (EMF 01), pois "essas 3 vias [...] são a chave do futuro [...] para esse trabalho de sucesso" (EMF 04) e "[...]para oferecer melhores condições a seus integrados" (EMF 07, CLI 02). Ainda, “[...]para garantir a continuidade do [...] negócio, que é um setor bastante controverso e com Legislação atuante" (FOR 01) e "[...]para continuar [...] a parceria entre produtor e empresa [...]" (PRO 02). Para tanto, acerca do compromisso da empresa com sustentabilidade, tendo em vista a cadeia de suprimentos, afirmaram os entrevistados que aquela tem por propósito assegurar os melhores produtos (EMF 01, FOR 03), com qualidade (EMF 02, FOR 01, FOR 03, PRO 04, PRO 05), rastreabilidade (EMF 02), comprometimento (FOR 02, PRO 05) respeitando a legislação (EMF 03, FOR 01) e as regulamentações (EMF 07), tendo em vista fazer negócios éticos por meio de parcerias (CLI 01) e ser referência na gestão responsável da sustentabilidade (CLI 02).

A comunicação das estratégias de sustentabilidade ocorre por meio de comunicados, emails, treinamentos de Compliance, intranet, portal via aplicativo no celular" (EMF 01), reuniões formais (CLI 01, FOR 02) e "materiais corporativos" (CLI 01). Por meio de "condições estabelecidas bem como [...] especificação técnica do produto" (FOR 01), ainda fomentada na orientação técnica (PRO 01, PRO 02, PRO 03, PRO 04, PRO 05, PRO 06). O monitoramento das ações estratégicas voltadas à sustentabilidade da cadeia de suprimentos se efetiva tendo por referência o "Índice Dow Jones de Sustentabilidade [...], Programa Thrive (Prosperar) [...] e o STP (Sustainable Tobacco Production) [...]" (EMF 01). Além disso, por meio de "[..]instrumentos desenvolvidos especificamente para tais fins, como pesquisa de satisfação" (EMF 02), por meio de auditórias e certificações tanto internas com externas (EMF 08, CLI 01, CLI 02, FOR 01, FOR 02) por meio do orientador agrícola junto aos produtores (PRO01, PRO02, PRO03, PRO04, PRO05, PRO06).

A Empresa Focal procura assegurar que a sustentabilidade de toda a cadeia esteja presente nas suas estratégias, seus objetivos e suas metas corporativas negócios, por meio do "[...]Line of Sight, onde os objetivos e as metas são cascateados das diretorias para os gerentes, coordenadores e demais colaboradores, garantindo o cumprimento de todas as premissas de sustentabilidade" (EMF 01), ainda por meio da rastreabilidade (EMF 02, FOR 02, PRO 02, PRO 04, PRO 06) e "[...]assegurando que haja retroalimentação dos processos" (EMF 02), bem como definindo o modelo funcional de "[...]relacionamento, comunicação, operacionalização de processos, compartilhando os interesses [...] e alinhando suas ações aos objetivos, incentivando e investindo em seus stakeholders" (CLI 02).

\subsection{Gestão sustentável}

No desenvolvimento da Gestão Sustentável da Cadeia de Suprimentos, a Empresa Focal identifica os critérios e requisitos de sustentabilidade definidos pelos clientes e incorpora a informação nas suas estratégias organizacionais (EMF 01, CLI 02) por meio de benchmarking (EMF 04, EMF 06, CLI 01) e análise de mercado (EMF 07). Identificados os requisitos, a operacionalização depende das parcerias entre a empresa e seus clientes no sentido de alcançar os objetivos da sustentabilidade da cadeia de suprimentos. As entrevistas revelam que isso ocorre pelo rigor nas atitudes, diretrizes, comportamentos e ações para com o cliente (EMF 04, CLI 01). De acordo com EMF 02 “[...] as operacionalizações são estabelecidas formalmente onde as partes se comprometem e se submetem a auditorias para garantir a rastreabilidade e compliance alinhados aos objetivos de sustentabilidade". Não obstante, CLI 02 afirma que se dá por meio "[...]do grau de competitividade em qualidade e preço, com base em relacionamentos de longo prazo, alinhamento de políticas e condutas que visem promover o desenvolvimento socioeconômico". 
Importa aqui destacar o compromisso na formalização do vínculo entre empresa e clientes no sentido de compartilhar as responsabilidades sobre a sustentabilidade da cadeia de suprimentos. Este compromisso é expresso formalmente em cláusulas contratuais (EMF 02, EMF 04, EMF 05, CLI 01, CLI 02) e "[...]aplicação do STP que corresponde a uma ferramenta para operacionalizar a adoção e garantir o monitoramento e a evolução de boas práticas ligadas à sustentabilidade na produção de tabaco" (EMF 05). Dentro de sua cadeia de suprimentos, a Empresa Focal atribui os papeis ou responsabilidades visando a garantia da sustentabilidade da cadeia de suprimentos. Conforme é evidenciado pelas entrevistas, "[...]os papéis e responsabilidades são muito bem distribuídos internamente, seguindo as premissas de Compliance da organização" (EMF 01). Ainda, por meio "[...]da comunicação de seus valores e princípios de negócio; através de suas políticas e normas; através de sua liderança, que dá o exemplo, e de todas as pessoas que integram a organização" (EMF 05), bem como, "[...]zelando pelo princípio ético das pessoas e processos [...], procurando sempre a distribuição dos procedimentos e necessidades gerando envolvimento para toda cadeia" (FOR 02). No mesmo entendimento, colocou o entrevistado PRO 02 que "[...] ]existe um acompanhamento, é fiscalizado na medida do possível [...], eu acho importante essa auditoria" para "[...] se seguir as regras, se não a empresa corta a parceria" (PRO 04).

No mesmo intento, os produtores se posicionaram, como pode ser visto na fala de PRO 03 "Eles tão sempre em cima né? Eles tão vendo, olhando, tirando tempo pra ir ver aqui, ali, ir na lavoura". Também se vincula às auditorias abrangentemente pontuadas como se vê nas considerações de PRO 02 "[...]o orientador chama e tenta corrigir, para a gente trabalhar da forma legal [...]" tal fala, também corrobora a asseveração da capacitação e do acompanhamento efetivo como recursos empregados. Não obstante, na mesma esfera, tem-se o gerenciamento dos riscos econômicos e de disrupção de processos operacionais nas relações com os fornecedores, bem como, fomentação do desenvolvimento do fornecedor frente à demanda enquanto recurso observável, ao passo que, de acordo com a entrevista de FOR 01, tal seara se desenrola quando "[...]o parceiro é contatado para esclarecimentos e, em não se adequando às demandas apresentadas, o parceiro é excluído do fornecimento". No entendimento do PRO 03, o resultado é "[...]não tá no padrão dela, [...] não tem escolha, é fora".

Percebe-se, portanto, que todos os envolvidos na cadeia de suprimentos são monitorados e auditados por instrumentos internos e externos a fim de assegurar os objetivos estratégicos voltados à GSCS. Tal colocação é corroborada pela entrevista de EMF 04 sobre a questão das avaliações da empresa focal para com o cerne do desempenho, ao afirmar que é efetivada sim,

[...] em todas as visitas, o orientador faz uma miniauditoria ou minivistoria e fala sobre o assunto sustentabilidade com os produtores; como preza nos contratos, é permitido a feitura de vistorias e auditorias aos fornecedores, [...] em questão à qualidade do material, qualidade do preço praticado [...], como trabalham, agem e dirigem o negócio [...], além lógico do STP.

Fica assim destacado o papel da Empresa Focal na GSCS, mantendo não somente mecanismos de controle, mas também de suporte para os demais componentes. Desvios identificados recebem atenção e as soluções são trabalhadas conjuntamente. Este processo fica claro no posicionamento dos gestores da Empresa Focal: “[...]primeiramente avaliamos o problema e solicitamos um plano de ação para que ele seja resolvido o mais rápido possível. Havendo a reincidência ou o não cumprimento de prazo para a normalização da ocorrência, o contrato é terminado" (EMF 01). "Por meio de check-list interno, monitoramento e auditoria, identificando o risco em cada não-conformidade diagnosticada. Procura contribuir com o intuito de amenizar o impacto negativo, visando evitar problemas com o negócio" (EMF 03). 
Confirmado o desvio de conduta em termos de sustentabilidade, sanciona-se [...] a lei contratual, fazendo a devida reunião [...] tenta uma comunicação. Em caso negativo partese talvez para as [...] medidas mais radicais; mas em todos os casos é questão de avaliação do risco e de como foi esse desvio (EMF 04).

Apesar dos esforços da Empresa Focal no sentido de desenvolver a GSCS, é possível identificar alguns possíveis gargalos na cadeia de suprimentos do tabaco. Neste aspecto, a pesquisa revelou que, além de fatores conjunturais, tais como a instabilidade crescente da economia e a incerteza acrescentada pelas políticas regulatórias, que a principal GSCS é a falta de informação e transparência. Tal questão prepondera mediante os levantamentos efetivados junto aos clientes, fornecedores e produtores componentes da cadeia de suprimentos da Empresa Focal, haja vista que sobressaíram respostas sem posicionamento efetivo ou fomentado pelo desconhecimento de fato acerca do que se indagou, como, por exemplo, ao se questionar aos fornecedores como a empresa focal procura assegurar que a sustentabilidade de toda a cadeia esteja presente nas suas estratégias, objetivos e metas corporativas / de negócios, o entrevistado FOR 01 citou não saber responder, o mesmo se observou nas respostas de FOR 03 que respondeu "não sei". No mesmo sentido, estão as respostas decorrentes dos produtores.

\subsection{Discussões}

A Empresa Focal exerce influência em toda a cadeia de suprimentos e assim, estreita o relacionamento, integrando expectativas, desenvolvendo soluções e reduzindo riscos (Beske \& Seuring, 2014; Gouda \& Saranga, 2018). Portanto a GSCS depende da capacidade da Empresa Focal de agregar tanto estrategicamente quanto no sentido operacional, oportunizando sinergia intra e interorganizações (Al-Mudimigh, Zairi \& Ahmed, 2004; Jia, Gong \& Brown, 2019; Vandchali, Cahoon \& Chen, 2021).

Com um mercado dinâmico e competitivo, a Empresa focal é constantemente desafiada a observar e identificar tendências de mercado sob pena de exclusão, posto que se faz necessário rever estratégias ou objetivos. Conforme Mintzberg (2000, p. 274), a formação de estratégia é “[...]um espaço complexo: é um desígnio arbitrário, uma visão intuitiva e um aprendizado intuitivo; ela envolve transformação e também perpetuação". Das organizações empresariais cada vez mais se exige - por parte da sociedade - uma atuação alinhada às propostas do desenvolvimento sustentável e a pensar modelos de negócio, produtos e serviços que permitam ao cidadão suprir suas necessidades ao menor custo socioambiental (Elkington, 2012; Purvis, Mao \& Robinson, 2019).

Cabe salientar que a sustentabilidade envolve a atividade economicamente viável, socialmente justa e ecologicamente correta - o denominado Tripé da Sustentabilidade ou TBL (Elkington; 2012), sendo que, dentro desta esfera, todos os aspectos devem interagir de forma a satisfazer o sucesso de uma organização, sendo este mensurado não apenas por meio de variáveis financeiras, mas também pelo seu desempenho social e ambiental (Blasi, Caporin \& Fontini, 2018; Ho \& Lucianetti, 2021).

Neste mesmo entendimento, cabe ponderar que a Empresa Focal, por si só, não mantêm uma estrutura de sustentabilidade, haja vista que, para a gestão sustentável, se fazem preponderantes os demais elos da cadeia de suprimentos (Jia, Gong \& Brown, 2019). Acerca disso, importa considerar as relações para com os clientes e fornecedores, enquanto elos desta cadeia. Estas relações entre Empresa Focal, clientes e fornecedores (produtores rurais aqui incluídos), ocorrem com base em duas definições intercorrentes. A primeira, estabelece que o "[...]objetivo principal é integrar e gerenciar a origem, fluxo e controle de materiais por meio de uma perspectiva sistêmica ao longo de múltiplas funções e múltiplos níveis" (Monczka, Trent \& Handfield, 1998, p. 12). Já a segunda, engloba todas as atividades "[...] associadas com o fluxo e transformação de bens desde o estágio de matérias primas (extração) até o consumidor final, assim como os fluxos de informação associados" (Handfield \& Nichols, 1999, p. 17). Assim, a atual atenção voltada às cadeias de suprimentos está 
relacionada, principalmente, ao gerenciamento de processos mais eficientes, inovadores e sustentáveis (Schulz et al., 2016; Good \& Thorpe, 2020; Ho \& Lucianetti, 2021).

Não obstante, a GSCS conduziu à expansão das fronteiras contemplando processos não anteriormente entendidos à gestão da cadeia de suprimentos. Há oportunidades de competitividade empresarial advindas do alinhamento entre ações de responsabilidade social e estratégia organizacional (Schulz et al, 2016). De acordo com Khodakarami et al. (2015), como benefício da estruturação da gestão da cadeia de suprimentos tem-se a vantagem competitiva decorrente da gestão eficiente da cadeia integrada aos conceitos de sustentabilidade nas principais funções dos negócios (Simões-Coelho \& Figueira, 2021).

Sob a perspectiva da Empresa Focal, reside aqui o principal gargalos à sustentabilidade: a integração de funções (processos) de negócios por meio dos membros participantes da cadeia de suprimentos, conforme já apontado por Lambert e Cooper (2000). O sucesso de uma organização estaria atrelado a sua capacidade de integração da rede de relacionamentos com as demais organizações que compõem a cadeia. Para alcançar este objetivo, a comunicação é elemento preponderante.

Por outro lado, ficou evidente a preocupação da Empresa Focal com os processos avaliativos que buscam alternativas eficientes por meio do monitoramento do desempenho, identificando áreas carentes de ação (Chan \& Qi, 2003; Ho \& Lucianetti, 2021). Para garantir processos eficientes e a preservação da imagem da organização, se faz necessário o gerenciamento dos riscos econômicos e de disrupção dos processos operacionais fomentando-se o desenvolvimento do fornecedor de maneira a identificar possíveis perdas (Beske \& Seuring, 2014; Gouda \& Saranga, 2018; Chowdhury \& Quaddus, 2021).

Deste modo, cabe salientar que a transformação do gerenciamento da cadeia de suprimento em gestão sustentável cria pressão significativa à Empresa Focal para modificar as ações suas e de seus fornecedores visando atender necessidades de sustentabilidade (Schrettle, 2014). Com isso a Empresa Focal passa a concentrar a responsabilidade sobre a sustentabilidade de toda a extensão da cadeia de suprimentos, ao mesmo tempo procurando garantir benefícios e competitividade a longo prazo, contabilizando atividades ambiental e socialmente responsáveis (Jia, Gong \& Brown, 2019. Tais práticas desencadeiam maior eficiência de material, energia e inovação (Gunasekaran \& Spalanzani, 2012) melhorando o desempenho econômico das organizações e criando reputação corporativa da marca no mercado (Wang \& Sarkis, 2013; Zimon, Tyan, \& Sroufe, 2020).

\section{CONSIDERAÇÕES FINAIS}

A base da literatura sobre a Gestão Sustentável da Cadeia de Suprimentos - GSCS, destaca que os aspectos ambientais, sociais e econômicos (lógica do TBL) devem ser tratados na estratégia empresarial de forma integrada, com o envolvimento de todas os componentes da cadeia de suprimentos. Assim, é necessário ponderar sobre o papel da Empresa Focal no estabelecimento das ações estratégicas voltadas à sustentabilidade de seu processo produtivo e sua influência ao longo de toda a cadeia de suprimentos.

Foi justamente sobre este tópico que a pesquisa definiu seu objetivo de investigar a ação estratégica da Empresa Focal para o desenvolvimento da Gestão Sustentável da Cadeia de Suprimentos do tabaco em folha. Com este foco de investigação, o objetivo restou plenamente alcançado, sendo possível observar que as opiniões divergem no que diz respeito à visão e operacionalização da cadeia de suprimentos do tabaco, porém em sua unanimidade, os entrevistados ressaltam que o seu cultivo se dá, fundamentalmente, em razão da questão econômica. $\mathrm{O}$ aspecto ambiental analisado evidencia a existência de uma preocupação com o tema, mas há também divergências de opiniões quando se coloca em questão sobre as penalidades da Legislação ou da Empresa Focal, caso algum ponto não seja observado pelos seus supridores ao longo da cadeia do tabaco. 
Com relação a Empresa Focal pode-se salientar que a dicotomia existente entre meio ambiente e economia ainda é a visão que prevalece entre os agentes econômicos. De posse deste entendimento, percebe-se a necessidade em se posicionar o contexto a jusante e a montante em relação a cadeia produtiva do tabaco, visando interpor este direcionamento ao que se propõe à Empresa Focal, assim, questionou-se sobre a incorporação da lógica da sustentabilidade (TBL) nas estratégias interorganizacionais definidas pela Empresa Focal e voltadas à gestão sustentável da cadeia de suprimentos do tabaco em folha.

Em síntese, as evidências da pesquisa apontaram que a incorporação da lógica da sustentabilidade nas estratégias interorganizacionais definidas pela Empresa Focal e voltadas à gestão sustentável da cadeia de suprimentos do tabaco em folha decorrem primeiramente da motivação estratégica, aliada aos relacionamentos para com os clientes, fornecedores e produtores de tabaco. Cada ação da Empresa focal destina-se à asseveração do pensamento estratégico sustentável anteriormente ponderado por ela, considerando-se de forma interrelacionada a esfera econômica, social e ambiental, de forma que o processo produtivo como um todo, partindo do fornecimento até a entrega do produto final ao cliente, perpassa uma estrutura fomentada na pesquisa e no desenvolvimento de melhores práticas agrícolas, industriais e comerciais, estas sendo ainda implementadas e monitoradas no sentido de sua garantia, bem como estruturadas a partir de ações sociais e ambientais, por meio de programas e processos que levam em conta o bem-estar das pessoas e a preservação do meio ambiente. Estas são questões que a empresa Focal deve incorporar nas suas práticas de gestão da cadeia de suprimentos, exercendo seu papel de governança e liderança.

Evidentemente que a pesquisa desenvolvida apresenta algumas limitações, as quais não permitem a generalização dos seus resultados. Neste contexto, tendo sido empregado o estudo de caso como estratégia de pesquisa, as conclusões e recomendações apresentadas representam apenas a realidade da Empresa Focal analisada, de fato limitando a amplitude da pesquisa, sem, contudo, limitar contribuição dos resultados para orientar os gestores deste e de outros setores sobre a incorporação da lógica do TBL na gestão sustentável das cadeias de suprimentos.

As evidências destacadas pela pesquisa constituem-se contribuições referentes às premissas da sustentabilidade no contexto da gestão das cadeias de suprimento, especialmente, nas cadeias agrícolas, face a diversidade, amplitude, impacto e resultados. Tais evidências contribuem à extensa compreensão da cadeia produtiva do tabaco, suas mazelas intercorrentes e relacionamentos, alinhando-se à proposição da Empresa Focal frente à sustentabilidade da cadeia de suprimentos (complexa e ambígua), visto que, apesar do destaque na agricultura brasileira, não é bem-vista por parte da sociedade, sendo este um segmento controverso da atividade econômica.

Estas contribuições podem estimular a realização de novos estudos, de maneira que estes permitam o aprofundamento e a propagação das práticas gerenciais envolvendo a sustentabilidade das cadeias de suprimentos. Também se recomenda que estudos subsequentes abordem um maior e mais abrangente detalhamento das relações entre os elos das cadeias de suprimentos, promovendose uma análise comparativa entre diferentes segmentos econômicos.

\section{REFERÊNCIAS}

Ageron, B., Gunasekaran, A., \& Spalanzani, A. (2012). Sustainable supply management: an empirical study. International Journal of Production Economics, 140, 168-182,

Al-Mudimigh, A. S., Zairi, M., \& Ahmed, A. M. M. (2004). Extending the concept of supply chain: the effective management of value chains. International Journal of Production Economics. Massachusetts, 87, 309-320. 
Alves, A. P. F., Silva, M. E. \& Santos, J. G. (2018). Colaboração para a sustentabilidade: práticas de membros de uma cadeia de suprimentos do Rio Grande do Sul. Revista de Gestão Social e Ambiental - RGSA, 12(1), 02-20.

Ansari, Z. N., \& Kant, R. (2017). A state-of-art literature review reflecting 15 years of focus on sustainable supply chain management. Journal of Cleaner Production, 142(04), 2524-2543.

Aqueveque, C., Rodrigo, P., \& Duran, I. J. (2018). Be bad but (still) look good: Can controversial industries enhance corporate reputation through CSR initiatives? Business Ethics, 27(3), 222-237.

Baldassarre, F., \& Campo, R. (2016). Sustainability as a marketing tool: to be or to appear to be? Business Horizons, 59(4), 421-429.

Barbieri, J. C., \& Cajazeira, J. E. R. (2010). Responsabilidade social e empresarial: da teoria à prática. São Paulo: Saraiva.

Begnis, H. S. M., Arend, S. C., \& Alievi, R. M. (2017). Confiança, comportamento oportunista e quebra de contratos na cadeia produtiva do tabaco. Revista de Administração da Universidade Federal de Santa Maria, 10(5), 888-907.

Belas, J. et al. (2021). Corporate social responsibility and sustainability issues of small and medium-sized enterprises. Corporate Social Responsibility Environment Management, 28, 721730.

Beske, P., \& Seuring, S. (2014). Putting sustainability into supply chain management. Supply Chain Management. An International Journal, 19(3), 322-331.

Blasi, S., Caporin, M., \& Fontini, F. (2018). A Multidimensional Analysis of the Relationship Between Corporate Social Responsibility and Firms' Economic Performance. Ecological Economics, 147, 218-229.

Bossel, H. Viability and sustainability. (1987). Matching development goals to resource constraints. Futures, 19(2), 114-128.

Brundtland, G. H. (1987). Our common future: call for action. Environmental Conservation, 14(4), 291-294.

Carter, C. R., \& Rogers, D. S. (2008). A framework of sustainable supply chain management: moving toward new theory. International Journal of Physical Distribution \& Logistics Management, 38(5), 360-387.

Carroll, A. B. (1999). Corporate social responsibility: evolution of a definitional construct. Business \& Society, 38(3), 268-295.

Carvalho, A. P., \& Barbieri, J. C. (2013). Inovações socioambientais em cadeias de suprimento: um estudo de caso sobre o papel da Empresa Focal. Revista da Administração e Inovação, 10(01), 232256.

Chan, F. T. S., \&; Qi, H. J. (2003). An innovative performance measurement method for supply chain management. International Journal Supply Chain Management, 8(3/4), 209-223.

Chang, C. W., Chiang, D. M., \& Pai, F. Y. (2012). Cooperative strategy in supply chain networks. Industrial Marketing Management, 41(7), 1114-1124. 
Chia, R. (2014). Reflections on the distinctiveness of European management scholarship. European Management Journal, 32(05), 683-688.

Chowdhury, M. M. H., \& Quaddus, M. A. (2021). Supply chain sustainability practices and governance for mitigating sustainability risk and improving market performance: a dynamic capability perspective. Journal of Cleaner Production, 278, 1-17.

Christopher, M. (2007). Logística e gerenciamento da cadeia de suprimentos. São Paulo: Thomson.

Cooper, M. C., Lambert, D. M., \& Pagh, J. D. (1997). Supply chain management: more than a new name for logistics. The International Journal of Logistics Management, 8(1), 1-14.

Cooper, M. C., Lambert, D. M., \& Pagh, J. D. (1998). Supply chain management: implementation issues and research opportunities. The International Journal of Logistics Management, 9(2), 1-19.

Daly, H. E. (1996). Beyond growth: the economics of sustainable development. Boston Beacon Press.

De Roeck, K., \& Delobbe, N. (2012). Do environmental CSR initiatives serve organizations' legitimacy in the oil industry? Exploring employees' reactions through organizational identification theory. Journal of Business Ethics, 110(4), 397-412.

Du, S. \& Vieira, E. T. (2012). Striving for legitimacy through corporate social responsibility: Insights from oil companies. Journal of Business Ethics, 110(4), 413-427.

Elkington, J. (1994). Towards the sustainable corporation: win-win-win business strategies for sustainable development. California Management Review, 1, p. 90-100.

Elkington, J. (2012). Sustentabilidade: canibais com garfo e faca. São Paulo: M. Books do Brasil Editora Ltda.

Erekson, H. O., Loucks, O. L., \& Aldag, C. (1994). The Dimensions of sustainability for business. American Journal of Business, 9(2), 3-6.

Fargnoli, M., Costantino, F., Di Gravio, G., \& Tronci, M. (2018). Product service-systems implementation: a customized framework to enhance sustainability and customer satisfaction. Journal of Cleaner Production, 188, 387-401.

Good, J. \& Thorpe, A. (2020). The nature of organizing: a relational approach to understanding business sustainability. Organization \& Environment, 33(3), 359-383.

Gouda, S. K., \& Saranga, H. (2018). Sustainable supply chains for supply chain sustainability: impact of sustainability efforts on supply chain risk. International Journal of Production Research, 56(17), 5820-5835.

Grougiou, V., Dedoulis, E., \& Leventis, S. (2016). Corporate social responsibility reporting and organizational stigma: the case of "sin" industries. Journal of Business Research, 69(2), 905-914.

Gunasekaran, A., \& Spalanzani, A. (2012). Sustainability of manufacturing and services: Investigations for research and applications. International Journal of Production Economics, 140(1), 35-47. 
Halldórsson et al., (2009). Complementary theories to supply chain management. Supply Chain Management an International Journal, 12(4), 284-296.

Handfield, R. B., \& Nichols Jr, L. (1999). Introduction to supply chain management. Upper Saddle River: Prentice Hall.

Ho, J., Lu, C., \& Lucianetti, L. (2021). Does engaging in corporate social responsibility activities influence firm performance? The moderating effects of risk preferences and performance measurement systems. Management Decision, 59(13), 15-37.

Hoffman, A. J. (2018). The next phase of business sustainability. Stanford Social Innovation Review, 16(2), 34-39.

Jia, F., Gong, Y., \& Brown, S. (2019). Multi-tier sustainable supply chain management: The role of supply chain leadership. International Journal of Production Economics, 217, 44-63.

Khan, S. A. R. et al, (2020). A state-of-the-art review and meta-analysis on sustainable supply chain management: Future research directions. Journal of Cleaner Production, 278, 1-12.

Khodakarami, F., Petersen, A., \& Venkatesan, R. (2015). Developing donor relationships: the role of the breadth of giving. Journal of Marketing, 79(4), 77-93.

Koberg, E., \& Longoni, A. (2019). A systematic review of sustainable supply chain management in global supply chains. Journal of Cleaner Production, 207, 1084-1098.

Lambert, D., \& Cooper, M. (2000). Issues in supply chain management. Industrial Marketing Management, 29, 65-83.

Lambert, D. M., Cooper, M. C., \& Pagh, J. D. (1998). Supply chain management: implementation issues and research opportunities. The International Journal of Logistics Management, 9(2), 1-20.

Lee, M.D. P. (2008). A review of the theories of corporate social responsibility: its evolutionary path and the road ahead. International Journal of Management Reviews, 10(1), 53-73.

Lis, A., Sudolska, A., \& Tomanek, M. (2020). Mapping research on sustainable supply-chain management. Sustainability, 12(10), 1-26.

Maixel, M. J., \& Luoma, P. (2015). Stakeholder pressure in sustainable supply chain management: a systematic review. International Journal of Physical Distribution \& Logistics Management, 45(1), 69-89.

Marcus, J., \& Roy, J. (2019). In search of sustainable behaviour: the role of core values and personality traits. Journal of Business Ethics, 158(1), 63-79.

McWilliams et al., (2016). Strategic decision making for the triple bottom line. Business Strategy and the Environment, 25(3), 193-204.

Mintzberg, H. (2000). Safári de estratégia; (2 $2^{\text {nd }}$ ed.) Porto Alegre: Bookman.

Monczka, R., Trent, R., \& Handfield, R. (1998). Purchasing and supply chain management. $2^{\text {nd }}$ ed. Ohio: SouthWestern - Thomson Learning. 
Mozzato, A. R., \& Grzybovski, D. (2011). Análise de conteúdo como técnica de análise de dados qualitativos no campo da administração: potencial e desafios. Revista de Administração Contemporânea - RAC, 15(4), 731-747.

Palazzo, G., \& Richter, U. (2005). CSR business as usual? The case of the tobacco Industry. Journal of Business Ethics, 61(1), 387-401.

Polistina, K. (2018). Are neoliberalist behaviours reflective of bullying? New perspectives on influences on sustainability and global citizenship. Environment, Development and Sustainability, 20(1), 175-196.

Porter, M. E., \& Kramer, M. R. (2002). The competitive advantage of corporate philanthropy. Harvard Business Review Press, December, 56-68.

Purvis, B., Mao, Y., \& Robinson, D. (2019). Three pillars of sustainability: in search of conceptual origins. Sustainability Science, 14(3), 681-695.

Qorri, A., Mujkić, Z., \& Kraslawski, A. (2018). A conceptual framework for measuring sustainability performance of supply chains. Journal of Cleaner Production, 189, 570-584.

Rajeev, A. et al. (2017). Evolution of sustainability in supply chain management: a literature review. Journal of Cleaner Production, 162, 299-314.

Sajjad, A., Eweje, G. \& Tappin, D. (2020). Managerial perspectives on drivers for and barriers to sustainable supply chain management implementation: evidence from New Zealand. Business Strategy and the Environment, 29(2), 592-604.

Schaltegger, J., Hörisch, J. \& Freeman, R. E. (2019). Business cases for sustainability: a stakeholder theory perspective. Organization \& Environment, 32(3), 191-212.

Schrettle, A. (2014). Explaining firm's sustainability efforts and their impact on firm performance. International Journal of Production Economics, 147(2), 73-84.

Schulz et al., (2016). Developing competitive advantage using the triple bottom line: a conceptual framework. Journal of Business \& Industrial Marketing, 31(4), 449-458.

Seuring, S., \& Müller, M. (2008). From a literature review to a conceptual framework for sustainable supply chain management. Journal of Cleaner Production, 16(15), 1699-1710.

Shokri Kahi, V. S. et al. (2017). How to evaluate sustainability of supply chains? A dynamic network DEA approach. Industrial Management \& Data Systems, 117(9), 1866-1889.

Simões-Coelho, M. F. \& Figueira, A. R. (2021). Why do companies engage in sustainability? Propositions and a framework of motivations. BAR - Brazilian Administration Review, 18(2), 1-32.

Solana-Ibáñez, J., Caravaca-Garratón, M. \& Teruel-Sánchez, R. (2020). Stakeholder perception on corporate reputation and management efficiency: evidence from the Spanish Defence sector. Corporate Social Responsibility and Environmental Management, 27(5), 2381-2399.

Souza, M. T. S., \& Ribeiro, H. C. M. (2013). Environmental sustainability: a meta-analysis of production in Brazilian management journals. Revista de Administração Contemporânea, 17(3), 368-396. 
Srivastava, S. K. (2007). Green supply chain management: a state-of-the-art literature review. International Journal of Management Reviews, 9(1), 53-80.

Talamini, E., Pedrozo, E. A., \& Silva, A. L. (2005). Gestão da cadeia de suprimentos e a segurança do alimento: uma pesquisa exploratória. Gestão \& Produção, 12(1), 107-120.

Vandchali, H. R., Cahoon, S., \& Chen, S. L. (2021). The impact of power on the depth of sustainability collaboration in the supply chain network for Australian food retailers. International Journal of Procurement Management, 14(2), 165-184.

Vitari, C., \& David, C. (2017). Sustainable management models: innovating through Permaculture. Journal of Management Development, 36(1), 14-36.

Vollero, A. et al. (2019). Corporate social responsibility information and involvement strategies in controversial industries. Corporate Social Responsibility and Environmental Management, 26(1), $141-151$.

Wang, Z., \& Sarkis, J. (2013). Investigating the relationship of sustainable supply chain management with corporate financial performance. International Journal of Productivity and Performance Management, 62(8), 871-888.

Xiao, C. et al. (2019). Inside the buying firm: exploring responses to paradoxical tensions in sustainable supply chain management. Journal of Supply Chain Management, 55(1), 3-20.

Yin, R. K. (2015). Estudo de caso: planejamento e métodos. (5. Ed). Porto Alegre: Bookman.

Zimon, D., Tyan, J., \& Sroufe, R. (2020). Drivers of sustainable supply chain management: practices to alignment with unsustainable development goals. International Journal for Quality Research, 14(1), 219-236.

\section{Como citar:}

Baptista Junior, G., \& Begnis, H. S. M. (2021). Gestão Sustentável na Cadeia de Suprimentos da Indústria do Tabaco. Revista De Gestão Social E Ambiental, 15, e02722. https://doi.org/10.24857/rgsa.v15i1.2722 